\title{
Ueber eine geometrische Anwendung der complexen Multiplication der elliptischen Functionen.
}

\author{
(Von Herrn L. Kiepert in Freiburg i. Br.)
}

Wenn sich die Coordinaten einer Curve als elliptische Functionen darstellen lassen, deren Argument der Bogen dieser Curve ist, so hat sie mit dem Kreise die Eigenschaft gemein, dass sich die Theilung ihres Bogens in $n$ gleiche Theile auf die Auflösung algebraischer Gleichungen zurückführen lässt. Der Grund davon liegt bekanntlich in dem Additions- und Multiplicationstheorem der elliptischen Functionen.

Im Allgemeinen wird der Grad der aufzulösenden Gleichungen sehr hoch, wenn man nur von der einfachen Multiplication Gebrauch macht. Wenn dagegen die complexe Multiplication der elliptischen Functionen anwendbar ist, so führen Gleichungen von weit niedrigerem Grade zum Ziele. So ist es bereits bekannt, dass die Theilung des Lemniscatenbogens in $4 q+1$ gleiche Theile, - wenn $4 q+1$ eine Primzahl ist, - zurückgeführt werden kann auf die Lösung einer Gleichung $q^{\text {ten }}$ Grades, aus deren Wurzeln noch die Quadratwurzel zu ziehen ist.

Etwas ganz Aehnliches gilt für die Theilung einer Curve, die ich bereits in meiner Dissertation *) (Seite 22) als Beispiel angeführt habe. Hier lässt sich die Theilung in $6 q+1$ gleiche Theile ausführen durch Auflösung einer Gleichung $q^{\text {ten }}$ Grades, aus deren Wurzeln noch die Cubikwurzel zu ziehen ist. Dies soll in der folgenden Abhandlung gezeigt werden. Ich wende dabei eine Methode an, wie sie analog Herr Weierstrass bei der Fünf-Theilung des Lemniscatenbogens in seiner Vorlesung (Sommer 1869) gegeben hat.

Während die Gleichung der Lemniscate am einfachsten in der Form $r^{2}=\cos 2 \varphi$ geschrieben wird, hat die zu behandelnde Curve die Gleichung

$$
r^{3}=\cos 3 \varphi,
$$

wobei $r$ der Radiusvector und $\varphi$ der Winkel ist, den der Radiusvector mit der festen Anfangsaxe bildet. Bezeichnen wir mit $u$ den Curvenbogen, so ist

*) De curvis quarum arcus integralibus ellipticis primi generis exprimuntur. Berolini, MDCCCLXX. Calvary. 
oder wenn wir $r^{2}=\frac{1}{\varrho}$ setzen, so wird

$$
d u=\sqrt{d r^{2}+r^{2} d \varphi^{2}}=\frac{d r}{\sqrt{1-r^{6}}}
$$

$$
d u=-\frac{d \varrho}{\sqrt{4 \varrho^{3}-4}} ; \quad u=-\int_{\infty} \frac{d \varrho}{\sqrt{4 \varrho^{3}-4}} .
$$

Der Bogen ist also ein elliptisches Integral erster Gattung, und $\varrho$ ist eine elliptische Function des Argumentes $u$. Setzen wir daher $\varrho=\wp u$, so ist

$$
\left(\frac{d \wp u}{d u}\right)^{2}=\wp^{\prime 2} u=4 \wp^{3} u-4,
$$

und zwar ist $\varrho$ genau eine $\wp-$ Function, wie sie Herr Weierstrass in seinen Vorlesungen eingeführt hat. Obgleich diese $\wp$-Function schon mehrfach in Dissertationen besprochen ist, will ich doch hier noch einmal ihre Eigenschaften, so weit ich sie in dem Folgenden benutze, kurz anführen.

Wird nämlich eine elliptische Function $z$ des Argumentes $u$ durch die Gleichung

$$
\left(\frac{d z}{d u}\right)^{2}=A z^{4}+4 B z^{3}+6 C z^{2}+4 B^{\prime} z+A^{\prime}
$$

definirt, so lässt sich stets eine Function $\wp u$ finden, die definirt ist durch die Gleichung

$$
\left(\frac{d \wp}{d u}\right)^{2}=4 \wp^{3}-g_{2 \wp}-g_{3},
$$

wo $g_{2}$ und $g_{3}$ die Invarianten zweiten und dritten Grades der biquadratischen Form

$$
A z^{4}+4 B z^{3}+6 C z^{2}+4 B^{\prime} z+A^{\prime}
$$

sind. Diese Function $\wp u$ ist eine gerade Function von $u$, die nur für Werthe von $u$, welche der Null congruent sind, unendlich wird. Dabei lässt sich $\wp u$ rational ausdrücken durch $z$ und $\frac{d z}{d u}$, und umgekehrt lässt sich $z$ rational ausdrücken durch $\wp u$ und $\frac{d \wp u}{d u}$.

Die Fundamentalperioden der elliptischen Function mögen $2 \omega_{1}$ und $2 \omega_{3}$ heissen, und es möge gesetzt werden:

wobei

$$
\wp \omega_{1}=e_{1}, \quad{ } \omega_{2}=e_{2}, \quad \wp \omega_{3}=e_{3},
$$

ist, dann wird

$$
\omega_{2}=-\omega_{1}-\omega_{3}
$$

$$
\begin{aligned}
\left(\frac{d \wp u}{d u}\right)^{2}=\wp^{\prime 2} u & =4\left(\wp u-e_{1}\right)\left(\wp u-e_{2}\right)\left(\wp u-e_{3}\right) \\
& =4 \wp^{3} u-g_{2} \wp u-g_{3} .
\end{aligned}
$$


Kiepert, Curventheilung durch complexe Multiplication elliptischer Functionen. 307

Die Function $\wp u$ ist abgeleitet aus einer anderen Function $\sigma u$ durch die Gleichung

$$
\frac{d^{2} \log \sigma u}{d u^{2}}=-\wp u \text {. }
$$

Umgekehrt können wir mit Hülfe dieser Gleichung die Function $\sigma u$ aus $\wp u$ ableiten, wenn wir zur Bestimmung der Integrationsconstanten die Gleichungen hinzufügen :

$$
\sigma(0)=0, \quad \sigma^{\prime}(0)=1, \quad \sigma^{\prime \prime}(0)=0 .
$$

$\sigma u$ ist eine ungerade Function und verschwindet nur für Werthe von $u$, die der Null congruent sind, also für

$$
u=2 \mu \omega_{1}+2 \nu \omega_{3},
$$

wo $\mu$ und $\nu$ beliebige ganźe Zahlen sind. Dagegen giebt es im Endlichen keinen Werth von $u$, für den $\sigma u$ unendlich wird.

Diese Function $\sigma u$ ist zwar selbst nicht periodisch, wenn man aber $u$ um irgend eine Periode $2 \mu \omega_{1}+2 \nu \omega_{3}$ vermehrt, so geht sie in sich selbst über, multiplicirt mit einem Exponentialfactor, dessen Exponent eine lineare Function des Argumentes $u$ ist. Am deutlichsten ersieht man diese Eigenschaft aus der Gleichung

wo

$$
\sigma\left(u+2 \mu \omega_{1}+2 \nu \omega_{3}\right)=(-1)^{\mu \nu+\mu+v} e^{\left(2 \mu \eta_{1}+2 v \eta_{3}\right)\left(u+\mu \omega_{1}+v\left(\omega_{3}\right)\right.} \sigma u,
$$

$$
\eta_{1}=\frac{\sigma^{\prime} \omega_{1}}{\sigma \omega_{1}}, \quad \eta_{3}=\frac{\sigma^{\prime} \omega_{3}}{\sigma \omega_{3}} .
$$

Die Function $\sigma u$ ist nicht von $u$ allein abhängig, sondern auch von den Grössen $g_{2}$ und $g_{3}$, die den Modul der elliptischen Function bestimmen. Wir werden daher für $\sigma u$ schreiben $\sigma\left(u, g_{2}, g_{3}\right)$, wenn es auf die Beschaffenheit der Grössen $g_{2}$ und $g_{3}$ ankommt.

In unserm Falle ist

$$
\begin{gathered}
g_{2}=0, \quad g_{3}=4 ; \\
e_{1}={ }_{\jmath} \omega_{1}=\varepsilon, \quad e_{2}={ }_{\S} \omega_{2}=1, \quad e_{3}={ }_{\wp} \omega_{3}=\varepsilon^{2},
\end{gathered}
$$

wobei $\varepsilon$ und $\varepsilon^{2}$ dritte Wurzeln der Einheit sind. Die beiden Fundamentalperioden $2 \omega_{1}$ und $2 \omega_{3}$ sind complex conjugirte Grössen und $2 \omega_{2}=-2 \omega_{1}-2 \omega_{3}$ ist die kleinste reelle Periode. Wir wollen zunächst diese Perioden bestimmen.

Aus den Eigenschaften der Function $\sigma u$ lässt sich folgende Relation herleiten

$$
\sigma\left(m u, g_{2}, g_{3}\right)=m \sigma\left(u, m^{4} g_{2}, m^{6} g_{3}{ }^{\prime},\right.
$$


308 Kiepert, Curventheilung durch complexe Multiplication elliptischer Functionen.

wo $m$ eine ganz beliebige Grösse ist. Durch logarithmische Differentiation folgt hieraus

$$
\begin{aligned}
& \left.\frac{\sigma^{\prime}\left(m u, g_{2}, g_{3}\right)}{\sigma\left(m u, g_{2}, g_{3}\right)}=\frac{1}{m} \frac{\sigma^{\prime}\left(u, m^{4} g_{2}, m^{6} g_{3}\right)}{\sigma\left(u, m^{4} g_{2},\right.}, m^{6} g_{3}\right) \\
& \wp\left(m u, g_{2}, g_{3}\right)=\frac{1}{m^{2}} \wp\left(u, m^{4} g_{2}, m^{6} g_{3}\right) .
\end{aligned}
$$

Wenn wir für unsern Fall $m$ gleich $\varepsilon$ setzen, so wird

$$
\sigma(\varepsilon u, 0,4)=\varepsilon \sigma(u, 0,4) \text {. }
$$

Da sich hier die Grössen $g_{2}$ und $g_{3}$ gar nicht ändern, so können wir kurz schreiben

$$
\begin{aligned}
\text { (1.) } \quad \sigma \varepsilon u & =\varepsilon \sigma u, \\
\text { (2.) } \quad \frac{\sigma^{\prime} \varepsilon u}{\sigma \varepsilon u} & =\varepsilon^{2} \frac{\sigma^{\prime} u^{\prime}}{\sigma u}, \\
\text { (3.). } \quad \wp \varepsilon u & =\varepsilon_{\wp} \dot{ } u
\end{aligned}
$$

Es war aber

$$
u=-\int_{\infty} \frac{d \wp u}{\sqrt{4 \wp^{3} u-4}},
$$

daraus folgt, dass die kleinste reelle halbe Periode

(4.) $\quad \omega_{2}=-\int_{\infty}^{1} \frac{d \wp u}{\sqrt{4 \wp^{3} u-4}}=\int_{\varepsilon}^{\varepsilon^{2}} \frac{d \wp u}{\sqrt{4 \wp^{3} u-4}}$.

Wenn wir jetzt $\varepsilon u$ statt $u$ setzen, so wird $\varepsilon \wp u$ aus $\wp u$, wir haben also

$$
\text { (5.) } \quad \omega_{2}=\varepsilon \int_{\varepsilon^{2}}^{1} \frac{d \wp u}{\sqrt{4 \wp^{3} u-4}}=\varepsilon \omega_{3}
$$

und ebenso

$$
\text { (6.) } \quad \omega_{2}=\varepsilon^{2} \omega_{1}
$$

oder

$$
\text { (7.) } \omega_{1}=\varepsilon \omega_{2}, \quad \omega_{3}=\varepsilon^{2} \omega_{2}, \quad \omega_{3}=\varepsilon \omega_{1} \text {. }
$$

Daraus folgt:

Jetzt sei

$$
\text { (8.) }\left\{\begin{array}{c}
\eta_{1}=\frac{\sigma^{\prime} \omega_{1}}{\sigma \omega_{1}}=\frac{\sigma^{\prime} \varepsilon \omega_{2}}{\sigma \varepsilon \omega_{2}}=\varepsilon^{2} \frac{\sigma^{\prime} \omega_{2}}{\sigma \omega_{2}}=\varepsilon^{2} \eta_{2} \\
\eta_{3}=\frac{\sigma^{\prime} \omega_{3}}{\sigma \omega_{3}}=\frac{\sigma^{\prime} \varepsilon^{2} \omega_{2}}{\sigma \varepsilon^{2} \omega_{2}}=\varepsilon \frac{\sigma^{\prime} \omega_{2}}{\sigma \omega_{2}}=\varepsilon \eta_{2} \\
\eta_{3}=\varepsilon^{2} \eta_{1} .
\end{array}\right.
$$

$$
\begin{gathered}
m=\alpha+\beta \varepsilon, \quad m^{\prime}=\alpha+\beta \varepsilon^{2}, \\
m m^{\prime}=n=\alpha^{2}+\beta^{2}-\alpha \beta
\end{gathered}
$$


Kiepert, Curventheilung durch complexe Multiplication elliptischer Functionen. 309

und

$$
\text { (9.) } \varphi(u)=\frac{\sigma m u}{\sigma^{n} u},
$$

dann hat diese Function $\varphi(u)$ die Perioden $2 \omega_{1}$ und $2 \omega_{3}$, wie wir sogleich beweisen wollen. Es ist:

$$
\begin{aligned}
\sigma m\left(u+2 \omega_{1}\right) & =\sigma\left(m u+2 m \omega_{1}\right) \\
& =\sigma\left(m u+2 \alpha \omega_{1}+2 \beta \omega_{3}\right) \\
& =(-1)^{\alpha \beta+\alpha+\beta} e^{\left(2 \alpha \eta_{1}+2 \beta \eta_{3}\right)\left(m u+\alpha \omega_{1}+\beta \omega_{3}\right)} \sigma m u .
\end{aligned}
$$

Nun ist aber

und

$$
2 \alpha \eta_{1}+2 \beta \eta_{3}=\left(\alpha+\beta \varepsilon^{2}\right) 2 \eta_{1}=2 m^{\prime} \eta_{1}
$$

folglich ist

$$
m u+\alpha \omega_{1}+\beta \omega_{3}=m u+\omega_{1}(\alpha+\beta \varepsilon)=m\left(u+\omega_{1}\right),
$$

(10.) $\sigma m\left(u+2 \omega_{1}\right)=(-1)^{\alpha \beta+\alpha+\beta} e^{2 n \eta_{1}\left(u+\omega_{1}\right)} \sigma m u$.

Vermehren wir im Nenner $u$ um $2 \omega_{1}$, so folgt aus

$$
\sigma\left(u+2 \omega_{1}\right)=-e^{2 \eta_{1}\left(u+\omega_{1}\right)} \sigma u
$$

also

$$
\sigma^{n}\left(u+2 \omega_{1}\right)=(-1)^{n} e^{2 n \eta_{1}\left(u+\omega_{1}\right)} \sigma^{n} u \text {, }
$$

$$
\varphi\left(u+2 \omega_{1}\right)=(-1)^{\alpha \beta+\alpha+\beta+n} \varphi(u) .
$$

Da aber $n=\alpha^{2}+\beta^{2}-\alpha \beta$, so wird

$$
\alpha \beta+\alpha+\beta+n=\alpha^{2}+\alpha+\beta^{2}+\beta,
$$

und dies ist stets eine gerade Zahl, da $\alpha^{2}+\alpha$ und $\beta^{2}+\beta$ einzeln gerade Zahlen sein müssen. Wir haben also

$$
\text { (12.) } \varphi\left(u+2 \omega_{1}\right)=\varphi(u) \text {. }
$$

Eben so lässt sich zeigen, dass

$$
\text { (13.) } \varphi\left(u+2 \omega_{3}\right)=\varphi(u) \text {. }
$$

Wir wollen jetzt untersuchen, für welche Werthe von $u$ diese elliptische Function unendlich und für welche Werthe sie gleich Null wird. Da $\sigma u$ mit $u$ zugleich verschwindet, so wird der Zähler für $u=0$ unendlich klein von der ersten Ordnung, der Nenner aber unendlich klein von der $n^{\text {ten }}$ Ordnung, es wird also für $u=0$ die Function $\varphi(u)$ unendlich gross von $\operatorname{der}(n-1)^{\text {ten }}$ Ordnung, und ausserdem giebt es keine Werthe von $u$, welche $\varphi(u)$ unendlich gross machen und $u=0$ nicht congruent sind.

Daraus folgt, dass $\varphi(u)$ auch genau für $n-1$ nicht congruente Werthe 
310 Kiepert, Curventheilung durch complexe Multiplication elliptischer Functionen.

von $u$ gleich Null wird; diese Werthe seien $u_{1}, u_{2}, \ldots u_{n-1} . \varphi(u)$ kann aber nur verschwinden, wenn der Zähler $\sigma m u$ verschwindet, wenn also $m u=2 k \omega_{1}+2 l \omega_{3}$ wird. Wir haben daher

oder

$$
m u_{\lambda}=2 k \omega_{1}+2 l \omega_{3},
$$

$$
\begin{aligned}
& m m^{\prime} u_{\lambda}=n u_{\lambda}=m^{\prime}\left(2 k \omega_{1}+2 l \omega_{3}\right), \\
& n u_{\lambda}=\left(\alpha+\beta \varepsilon^{2}\right)\left(k \varepsilon+l \varepsilon^{2}\right) 2 \omega_{2} \\
& =2 \omega_{2}\left\{\alpha\left(k \varepsilon+l \varepsilon^{2}\right)+\beta(k+l \varepsilon)\right\} .
\end{aligned}
$$

Bezeichnen wir mit $u_{\lambda}^{\prime}$ die zu $u_{\lambda}$ conjugirt complexe Grösse, so ist

$$
\begin{aligned}
n u_{\lambda}^{\prime} & =2 \omega_{2}\left\{\alpha\left(k \varepsilon^{2}+l \varepsilon\right)+\beta\left(k+l \varepsilon^{2}\right)\right\}, \\
n\left(u_{\lambda}+u_{\lambda}^{\prime}\right) & =2 \omega_{2}\{\alpha(-k-l)+\beta(2 k-l)\} .
\end{aligned}
$$

Jetzt nehmen wir an, $n$ sei eine Primzahl, dann müssen $\alpha$ und $\beta$ relativ prim sein, denn jeder Factor, den sie gemeinsam haben, tritt in $n$ als quadratischer Factor auf. Wir können deshalb 2 Zahlen $\gamma$ und $\delta$ finden, so dass

$$
\alpha \delta+\beta \gamma=1 \text {. }
$$

$k$ und $l$ sind ganz beliebige Zahlen, deshalb können wir setzen

$$
\text { (15.) } \quad k=\lambda(\gamma-\delta), \quad l=\lambda(-\gamma-2 \delta) \text {. }
$$

Tragen wir diese Werthe in Gleichung (14.) ein, so kommt

$$
\begin{aligned}
n\left(u_{\lambda}+u_{\lambda}^{\prime}\right) & =2 \omega_{2}\{3 \lambda \alpha \delta+3 \lambda \beta \gamma\} \\
& =6 \lambda \omega_{2}(\alpha \delta+\beta \gamma)=6 \lambda \omega_{2} .
\end{aligned}
$$

Wenn wir $k$ und $l$ diese Werthe geben und $\lambda$ die Werthe von 1 bis $n-1$ durchlaufen lassen, so erhalten wir $n-1$ Werthe $u_{\lambda}$, für welche $\varphi\left(u_{\lambda}\right)$ verschwindet; es ist nur noch zu zeigen, dass sie ein vollständiges System incongruenter Werthe von $u$ darstellen, für welche $\varphi(u)$ verschwindet. Es ist

$$
u_{1}+u_{1}^{\prime}=\frac{3}{n} 2 \omega_{2}, \quad u_{2}+u_{2}^{\prime}=\frac{6}{n} 2 \omega_{2}, \ldots \quad u_{n-1}+u_{n-1}^{\prime}=\frac{3 n-3}{n} 2 \omega_{2} .
$$

Wenn aber $n$ nicht durch 3 theilbar ist, - was stets der Fall ist, da $n$ eine Primzahl sein soll, - so sind diese $n$ Grössen in der That incongruent, folglich erst recht $u_{1}, u_{2}, \ldots u_{n-1}$. Da es aber überhaupt nur $n-1$ incongruente Werthe von $u$ giebt, für welche $\varphi(u)$ verschwindet, so repräsentiren $u_{1}$, $u_{2}, \ldots u_{n-1}$ ein vollständiges System incongruenter Werthe von $u$, für welche $\varphi(u)=0$ wird.

Zugleich ergiebt sich folgendes: Wenn wir $\wp u_{\lambda}$ aufgefunden haben, so 
haben wir auch sofort die conjugirte Grösse $\wp_{\lambda}^{\prime}$ und können daraus

$$
\wp\left(u_{\lambda}+u_{\lambda}^{\prime}\right)=\wp \frac{6 \lambda \omega_{2}}{n}
$$

ableiten, da ganz allgemein

$$
\wp(v+w)=\frac{(\wp v+\wp w)\left(2 \wp v \wp w-\frac{1}{2} g_{2}\right)-g_{3}-\wp^{\prime} v \wp^{\prime} w}{2(\wp v-\wp v)^{2}}
$$

ist. Wir können dann also unmittelbar die Grössen

$$
\wp \frac{6 \omega_{2}}{n}, \quad \wp \frac{12 \omega_{2}}{n}, \quad \ldots \quad \wp \frac{(6 n-6) \omega_{2}}{n}
$$

construiren, und da $\wp u=\frac{1}{r^{2}}$ ist, auch die zugehörigen Werthe von $r$. Nun war aber

$$
\begin{aligned}
\omega_{2} & =-\int_{\infty}^{1} \frac{d \wp}{\sqrt{4 \vartheta^{3}-4}} \\
& =\int_{0}^{1} \frac{d r}{\sqrt{1-r^{6}}},
\end{aligned}
$$

folglich ist $\omega_{2}$ der Bogen $O A$ und $6 \omega_{2}$ der Bogen der ganzen Curve. Sind also die angeführten Werthe des Radiusvector $r$ bekannt, so sind auch die gesuchten Theilpunkte auf der Curve gegeben.

Daher haben wir nur noch die Grössen $\wp u_{\lambda}$ zu bestimmen, und dies ist jetzt mit Hülfe eines Satzes, den Herr Weierstrass in seinen Vorlesungen gegeben hat, sehr leicht ausführbar. Dieser Satz lautet: „Ist $\varphi(u)$ eine gerade Function, die nur für $u$ gleich Null unendlich gross wird und zwar unendlich gross von der

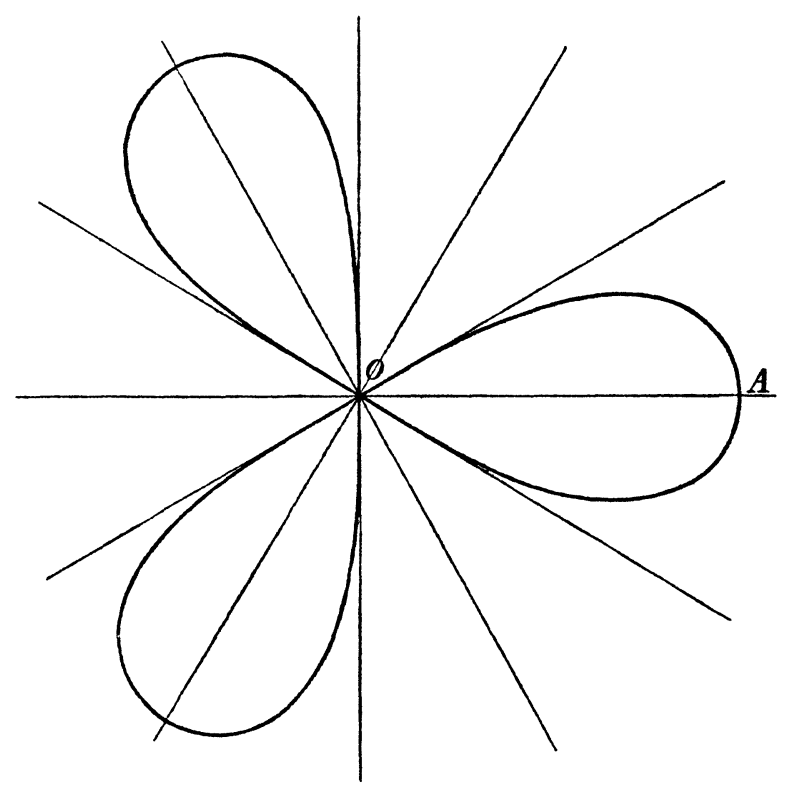
$(n-1)^{\text {ten }}$ Ordnung, so lässt $\operatorname{sich} \varphi(u)$ als ganze rationale Function von you darstellen, deren Grad $\frac{n-1}{2}$ ist.."

Die Voraussetzungen dieses Satzes passen auf unsere Function $\varphi(u)$, 
folglich können wir sie so darstellen. Setzen wir diese Function von $\wp u$ gleich Null, so erhalten wir eine Gleichung, deren Wurzeln die Grössen $\wp u_{\lambda}$ sind, weil die linke Seite $\varphi(u)$ für $u=u_{\lambda}$ verschwindet.

Da $\wp u$ eine gerade elliptische Function ist, so wird $\wp v=\wp w$ sein, wenn sich $v$ von $-w$ nur um eine ganze Periode unterscheidet. Dies ist der Grund, weshalb von den Grössen

$$
\wp u_{1}, \wp u_{2}, \ldots \varphi_{n-1}
$$

je zwei einander gleich werden, und dass die Anzahl der Wurzeln jener Gleichung sich auf $\frac{n-1}{2}$ reducirt.

Aus der Entwickelung von $\varphi(u)$ folgt, dass der Coefficient von $(\wp u)^{\frac{n-1}{2}}$ gleich $m$ wird. Die Bestimmung jener Gleichung von $\wp u$ wird noch wesentlich vereinfacht durch folgende Eigenschaft von $\varphi(u)$ : Wenn $n=\alpha^{2}+\beta^{2}-\alpha \beta$ eine von 3 verschiedene Primzahl ist, so hat $n$ stets die Form $6 q+1$, und umgekehrt lässt sich jede Primzahl von der Form $6 q+1$ auf die Form $\alpha^{2}+\beta^{2}-\alpha \beta$ bringen. Deshalb folgt aus der Gleichung $\sigma \varepsilon u=\varepsilon \sigma u$

$$
\varphi(\varepsilon u)=\frac{\varepsilon \sigma m u}{\varepsilon^{n} \sigma^{n} u}=\frac{1}{\varepsilon^{6 q}} \frac{\sigma m u}{\sigma^{n} u}=\varphi(u) .
$$

$\varphi(u)$ ändert sich also nicht, wenn wir $\varepsilon u$ statt $u$ setzen, folglich darf sich darn auch jene Function von $\wp u$ nicht ändern. Nun ist aber $\wp \varepsilon u=\varepsilon \wp u$, folglich darf nur der Cubus von øu vorkommen.

Somit ist bewiesen:

Wenn $n=6 q+1$ ist, so wird $\varphi(u)$ eine ganze rationale Function $q^{\text {ten }}$ Grades von $\wp^{3} u$, die wir leicht auffinden können, indem wir setzen

$$
\varphi(u)=m\left\{\wp^{3 q} u+A_{1} \vartheta^{3 q-3} u+A_{2} \vartheta^{3 q-6} u+\cdots+A_{q}\right\} .
$$

Entwickeln wir auf beiden Seiten nach Potenzen von $u$ und setzen die Coefficienten gleicher Potenzen einander gleich, so bestimmen sich daraus die Grössen $A_{1}, A_{2}, \ldots A_{q}$. Die Wurzeln der Gleichung

$$
\wp^{3 q}+A_{1 \vartheta^{3 q-3}}+A_{2} \wp^{3 q-6}+\cdots+A_{q}=0
$$

sind dann die gesuchten Grössen und geben die Lösung der allgemeinen Aufgabe.

Wir wollen zum Schluss noch die Grössen $A_{1}, A_{2}$ und $A_{3}$ wirklich berechnen. Dazu brauchen wir die Entwickelungen von $\sigma u$ und $\wp u$. Diese sind für $g_{2}=0$ und $g_{3}=4$

$$
\sigma u=u+a_{1} u^{7}+a_{2} u^{13}+a_{3} u^{19}+\cdots,
$$


Kiepert, Curventheilung durch complexe Multiplication elliptischer Functionen. 313

wobei

$$
a_{1}=\frac{-1}{2.3 .5 .7}, \quad a_{2}=\frac{-1}{2^{3} \cdot 3^{2} \cdot 5^{2} \cdot 7 \cdot 11.13}, \quad a_{3}=\frac{23}{2^{4} \cdot 3^{4} \cdot 5^{3} \cdot 7^{2} \cdot 11.13 .17 .19}, \quad \ldots ;
$$

ferner ist hier:

wobei

$$
\wp u=u^{-2}+b_{1} u^{4}+b_{2} u^{10}+b_{3} u^{16}+\cdots,
$$

$$
b_{1}=\frac{1}{7}, \quad b_{2}=\frac{1}{7^{2} .13}, \quad b_{3}=\frac{1}{7^{3} \cdot 13.19}, \ldots
$$

Daraus folgt

$$
\begin{aligned}
\sigma m u & =m\left\{u+a_{1} m^{6} u^{7}+a_{2} m^{12} u^{13}+a_{3} m^{18} u^{19}+\cdots\right\} \\
\sigma^{6 q+1} u= & u^{6 q+1}+(6 q+1) a_{1} u^{6 q+7}+(6 q+1)\left(a_{2}+3 q a_{1}^{2}\right) u^{6 q+13} \\
& \quad+(6 q+1)\left[a_{3}+6 q a_{1} a_{2}+q(6 q-1) a_{1}^{3}\right] u^{6 q+19}+\cdots
\end{aligned}
$$

und

$$
\begin{gathered}
\varphi(u)=\frac{\sigma m u}{\sigma^{6 q+1} u} \\
=m\left\{u^{-6 q}+\left(m^{6}-6 q-1\right) a_{1} u^{-6 q+6}+\left[\left(m^{12}-6 q-1\right) a_{2}-(6 q+1)\left(m^{6}-3 q-1\right) a_{1}^{2}\right] u^{-6 q+12}\right. \\
+\left[\left(m^{19}-6 q-1\right) a_{3}-(6 q+1)\left(m^{12}+m^{6}-6 q-2\right) a_{1} a_{2}+(6 q+1)\left\{m^{6}-1+\left(3 m^{6}-5\right) q-6 q^{2}\right\} a_{1}^{8}\right] u^{-6 q+18}+\ldots
\end{gathered}
$$

Ausserdem ist

$$
\begin{gathered}
\wp^{3 q} u+A_{1 \vartheta^{3 q-3}} u+A_{2} \wp^{3 q-6} u+\cdots+A_{q} \\
=u^{-6 q}+\left(3 q b_{1}+A_{1}\right) u^{-6 q+6}+\left[3 q\left(b_{2}+\frac{3 q-1}{2} b_{1}^{2}\right)+A_{1}(3 q-3) b_{1}+A_{2}\right] u^{-6 q+12} \\
+\left[3 q\left\{b_{3}+(3 q-1) b_{1} b_{2}+\frac{(3 q-1)(3 q-2)}{6} b_{1}^{3}\right\}+A_{1}(3 q-3)\left(b_{2}+\frac{3 q-4}{2} b_{1}^{2}\right)\right. \\
\left.+A_{2}(3 q-6) b_{1}+A_{3}\right] u^{-6 q+18}+\cdots .
\end{gathered}
$$

Aus der Vergleichung dieser Entwickelung mit der von $\varphi(u)$ ergeben sich sofort die Grössen $A_{1}, A_{2}, A_{3}, \ldots$

Für $n=7$ wird $m=3+\varepsilon, m^{\prime}=3+\varepsilon^{2}$;

dann ist

$$
q=1 \text {, }
$$

und hieraus folgt

$$
m^{6}=37+360 \varepsilon
$$

$$
A_{1}=\left(-7+m^{6}\right) a_{1}-3 b_{1}=-4 \frac{(1+3 \varepsilon)}{7}=\frac{-4}{1+3 \varepsilon^{2}} ;
$$

al so

$$
\begin{aligned}
\varphi(u) & =m\left\{\wp^{3} u-\frac{4}{1+3 \varepsilon^{2}}\right\}, \\
\wp u_{\lambda} & =\sqrt[3]{\frac{4}{1+3 \varepsilon^{2}}} .
\end{aligned}
$$

Journal für Mathematik Bd. LXXIV. Heft 4. 
314 Kiepert, Curventheilung durch complexe Multiplication elliptischer Function en.

Für $n=13$ setzen wir $m=4+\varepsilon, m^{\prime}=4+\varepsilon^{2}$;

$$
q=2 \text {. }
$$

Hier wird

und

$$
\begin{gathered}
m^{6}=1513+2520 \varepsilon \\
m^{12}=-4061231+1275120 \varepsilon \\
A_{1}=\left(m^{6}-13\right) a_{1}-6 b_{1}=-4(2+3 \varepsilon)
\end{gathered}
$$

$$
\begin{aligned}
A_{2} & =\left(m^{12}-13\right) a_{2}+13\left(7-m^{6}\right) a_{1}^{2}-6 b_{2}-15 b_{1}^{2}-3 b_{1} A_{1} \\
& =16 \cdot \frac{4+3 \varepsilon}{13}=\frac{16}{4+3 \varepsilon^{2}} .
\end{aligned}
$$

Daher werden für $n=13$ die Grössen $\wp u_{\lambda}$ die Wurzeln der Gleichung

$$
\wp^{6} u-4(2+3 \varepsilon) \wp^{3} u+\frac{16}{4+3 \varepsilon^{2}}=0 .
$$

In ähnlicher Weise findet man für die Theilung in 19 gleiche Theile die Gleichung dritten Grades

$$
\wp^{9} u+12(3+2 \varepsilon) \wp^{6} u-48(1+\varepsilon) \wp^{3} u+\frac{64}{5+3 \varepsilon}=0
$$

und für die Theilung in 31 gleiche Theile die Gleichung fünften Grades $\wp^{15} u-4(5-33 \varepsilon) \wp_{8}^{12} u+112(4+3 \varepsilon) \wp^{9} u-64(37+18 \varepsilon)_{\wp}{ }^{6} u+256(5+3 \varepsilon)_{\vartheta^{3}} u-\frac{1024}{1+6 \varepsilon}=0$.

Berlin, 23. März 1871. 